\title{
Personalized medicine takes foresight and smart public policies
}

\begin{abstract}
Governments and health care payers everywhere seek ways how to design public funding policies for high-cost drugs, which are increasingly personalized and often only effective in small population groups or sub-groups. Access to drugs is usually preceded by health technology assessment. Challenges for access to effective drugs at the patient level remain, as the current reimbursement environment is not ready to encompass the complexity of Personalized Medicine. A high level of uncertainty remains as to how, for whom and which type of Personalized Medicine should unfold in the future. Foresight modeling may come into play here. Foresight is a systematic approach to look into the longer-term future of science and technologies and their potential impacts on society. It aims at identifying research and development areas likely to generate future economic, environmental and social benefits.
\end{abstract}

Keywords: personalized medicine, financing, foresight
Volume 2 Issue 3 - 2015

\author{
Maria M Hofmarcher \\ Health System Intelligence, Austria
}

Correspondence: Maria M Hofmarcher, Health System Intelligence,A-I080 Wien, Josefstaedter Strasse 14/60,Austria, Tel 43 | 4022724,

Email maria.hofmarcher@healthsystemintelligence.eu

Received: January 19,2015 | Published: April 14, 2015
Abbreviations: PM, personalized medicine; QALY, quality adjusted life years

\section{Introduction}

Governments and health care payers everywhere seek ways how to design public funding policies for high-cost drugs, which are increasingly personalized and often only effective in small subgroups of the population. Recently, two targeted oncology therapies, Roche/Genentech's Zelboraf and Pfizer's Xalkori, were approved in conjunction with companion diagnostics. ${ }^{1}$ In Europe, an estimated number of thirteen drugs are approved for which diagnostic tests are either required or recommended. ${ }^{2}$ Currently, tailored cancer drugs appear to be the frontrunners in the area of Personalized Medicine (PM) and likely have the greatest impact on future resource allocation. ${ }^{3}$

Access to drugs is usually prepared by health technology assessments. After marketing authorization of a new drug many countries perform real world benefit assessments in order to determine whether a new drug deserves to be reimbursed by public payers, and at what price. This second assessment often compares the effectiveness of the new product against existing therapeutic options. Often, incremental cost per life year gained and incremental cost per QALY are applied to determine the reimbursement status. ${ }^{4}$ Still the challenges for access to effective drugs at the patient level need to be addressed, as the current reimbursement environment is not ready to embrace the complexity of PM. The European Science Fund (2012) defined PM as genomic, stratified, and precise, and as encompassing predictive, preventive, personalized, and participatory medicine, which is also called P4 medicine.

PM has its roots in the human genome sequencing in 2001. R \& D efforts in this area and molecular biology have produced technologies and techniques for use in life science research, including medicine. Medical technologies including drugs are the area with the highest number of patent filings between 1978 and 2011, and also in recent years the number of patented biotech and Pharma inventions has remained high, outnumbering patents in e.g. digital communication. ${ }^{5}$ However, it is estimated that 90 percent of drugs currently on the market work in only about 40 percent of individuals translating annually into \$350billion worth of ineffective prescriptions. ${ }^{1}$ Increased efficacy of treatment is thus required calling for regulatory policies that would complement measures aiming at combating over-prescription and under-consumption of drugs at the clinical level.

\section{Conclusion}

The promise of better targeted therapies involves

i. Enhanced efficacy leading to better health or improved quality of life for those who are identified to benefit,

ii. Associated cost savings and improved patient safety by preventing people from taking ineffective or even harmful medicines and

iii. The offset of high cost of tailored drugs by these cost savings

Still, stakeholders and decision-makers need much better evidence and HTA based guidance to take decisions on how, for whom and which type of PM should unfold in the future. Broadly this concerns three issues. First, regulators and public payers must determine to which degree diagnostic testing, investment in infrastructure for PM including training of professionals and incentives for the industry are public goods. If the answer is yes, this would involve investments in IT infrastructure e.g. biobanks, genome sequencing and bioinformatics to ensure test standards as well as appropriate data security and oversight. Second, governments need to address limitations of social health insurance to adequately allocate funding to PM. Generally, in pay-as-you-go systems, e.g. in Germany, France, or Austria, "average" patients can expect "average" benefits in exchange to a defined social health insurance contribution they pay according to their ability (vertical equity). Likewise, the principle of horizontal equity requires treatment according to need. For example, many inexpensive drugs are cost-effective on average but ineffective for a minority of patients for whom only a more expensive drug will work. Scarce health care budgets, increasingly competing with claims to bolster spending on chronic and social care imply that some people may remain "under-treated" when they fall ill of a disease for which only little resources remain available. 
Finally, progressive adoption of PM needs the support of a range of stakeholders and payers., ${ }^{2,6}$

Given the requirement to establish infrastructure and longterm development programs, including incentives for new industry business models, it will be essential to establish sustainable decisionmaking models to evaluate the potential, risks, and overall return on investment in PM. This implies that payers and administrators need to initiate a process that will enable them to identify areas where targeted early interventions would reduce disease burden and cost. Foresight modeling may come into play here. Foresight is a systematic approach to look into longer-term future of science and technologies and predict their potential impacts on society. It aims at identifying areas of scientific research and technological development likely to generate future economic, environmental and social benefits. Complementary to technology assessment, foresight modeling should enable the development of strategies for regulators and public payers who aim at ensuring social goals while encouraging innovation in and diffusion of PM based on need. Thus, a bundle of policy measures are needed to safeguard broad access to PM and the sustainability of public (health) finance. Governments and health leaders will need to

a. Embrace data, information, and a range of stakeholders to transform health and care as health care systems enter the age of PM.

b. Phase-in non-earmarked funding of the drug budget where pertinent to balance the funding of care needs in particular in pay-as-you-go systems.

c. Use foresight modeling to govern and enhance innovation in PM while strengthening the knowledge base for decision-making in order to reduce uncertainties and limit funding risks.

In particular, a scenario method could be applied. This is a foresight policy analysis tool that lays out a set of possible future conditions. At supranational, national, regional and local levels scenarios can be used to provide intelligence to strategic public policy decisions and guide investments. For example, scenarios allow new insights into the opportunities and risks involved in public health funding of PM. Such insights should foster the development of smart policies across government sectors and jurisdictions. And they should be driven by the policy goal to improve the health of the population further while ensuring the sustainability of future financing of high-quality health care for all people.

\section{Acknowledgements}

None.

\section{Conflict of interest}

The author declares no conflict of interest.

\section{References}

1. Nature biotechnology. What happened to personalized medicine? Nature biotechnology. 2012;30:1.

2. Branzen K. Personalized Medicine, a new era for health care and industry. Sweden: A futures study from Life Science Foresight Institute; 2013.

3. Jonsson B. Can society afford innovative cancer treatments? Europe: EU Press Dialog; 2012.

4. Paris V, Belloni A. Value in Pharmaceutical Pricing. OECD Health Working Papers. 2013;63:74.

5. World Health Organization (WHO), World Intellectual Property Organization (WIPO), World Trade Organization (WTO). Promoting access to medical technologies and innovations, Intersections between Public Health, Intellectual Property and Trade. Geneva: World Health Organization, World Intellectual Property Organization, World Trade Organization; 2013.

6. European Science Foundation (ESF). Personalized Medicine for the European Citizen-towards more precise medicine for the diagnosis, treatment and prevention of disease (iPM). Europe: European Science Foundation; 2012. 